\title{
Georg Weigel. Ein Beitrag zur Reformationsgeschichte Altpreussens und Lithauens.
}

\author{
Von D. Dr. Th. Wotsehke.
}

Dem Thorner Professor Hartknoch verdanken wir eine eingehende Kirchengeschichte Altpreußens. Vor mehr denn 200 Jahren geschrieben, ist sie noch heute, anf das Ganze gesehen, nicht uberhoit. Grundlich nnterrichtet sie anf 1100 Seiten uber alle kirchlichen Ereignisse bis zam Jahre 1680 . Aber mit keinem Worte erwähnt sie jenen unrahigen Theologen, der 1562/63 die kirchlichen Wirren in Königsberg noch mehrte, zum Osianderschen Hader einen Sakramentsstreit fugte, dann in Wilna und Lithauen aberhaupt verhängnisvoll wirkte und schließlich fast in die römische Kirche zurticktrat, Georg Weigel aus Nurnberg. Auch andere Kirchengeschichten wissen nichts von ihn. Nur Löscher „Historia motaum " kennt seinen Namen, bringt seine kurze Confession nnd einen Streitbrief seiner Feder wider Epplin, aber näberes weiB anch dieser Historiker uber ihn nicht zu berichten. Außer den beiden genannten Urkanden ist ihm von Weigel nichts bekannt; uber die Zeit seines Auftretens hat er nur eine Vermutung, und diese Vermutang ist falsch. Es wird deshalb die geschichtliche Forschung fördern, nähere Nachrichten über diesen fast ganz unbekannten Theologen zu bringen-

Justas Jonas, der Jüngere, der ungluckliche Sohn des treuen Freandes Lathers, schreibt in Beantwortang einest berzoglichen Briefes vom 14. Januar 1561 an den Hohenzoller in Königsberg ${ }^{1}$ ): „Es ist hier in Wittenberg ein frommer, gottesfurchtiger, eingezogener, stiller, ganz gelehrter Gesell mit Namen Magister Georg Weigel, ein Nurnberger, seines Alters angefähr ein- oder zweinnddreiBig Jahre, eine feine,

1) Vergl. J. Voigt, Briefwechsel der berühmtesten Gelehrten mit Herzog Albrecht von Preaßen S. 388. 
lange, ansehuliche, sittige Person, welcher drei Jahre von dem Rate zu Nürnberg allhier im Studium der Theologie verlegt ist, zuvor aber etliche Jahre allhier seinen Studien mit FleiB obgelegen. Ich habe ihu etliche Male hier in der Schloßkirche, wenn die verordneten Prädikanten verhindert gewesen, predigen hören, habe seiner zuvor ganz and gar keine Kunde gehabt, aus seinen Predigten aber gespurt, dab er nicht allein die Bucher, worans der mebrere Teil derjenigen, die sich für Theologen ausgeben, ihre Kanst schöpfen, sondern anch andere Schriften mit Fleiß gelesen und ein nicht gemeines Judizinm habe. Ich habe iba desbalb zu mir gebeten und nach der Länge ron den Artikeln, dartuber man itzo streitig ist, konferiert und befanden, da $B$ er dieselbigen Kontroverse allesamt aus dem Fundament versteht, so daß zu wünschen wäre, daß nnter denjenigen, welchen die Herde Gottes za weiden befohlen ist, viele seines Gleichen sein möchten. So hat er auf der Kanzel gar eine gate Art zn reden; damit aber E. F. D. eine kleine Anzeige habe, daß der Bericht, den ich von ihm toe, wahrhaftig sei, tuberschicke ich E. F. D. hierneben ein Buchlein welehes er gemacht und allhier vor einem Jahr im Drack hat ausgehen lassen. Ich sähe darum gern, daß dieser Maun in E. F. D. Dienst käme, weil ich keinen noch gehört habe, der uber die Kontrovers Osianders so recht judiaiert hätte als er. Er ist in Wahrheit ein groBer Theologus. So stimmt sein Judiziam mit allen recht verständigen Lenten darin uberein, daB er meint, es habe der mehre Teil den Osiander ex praeiudicio verdammt."

Der hier so warm Empfohlene war 1529 in Nünberg geboren, hatte die Schule seiner Vaterstadt besucht, dann das Straßbarger Gymnasium vier Jahre; am 29. Juni 154\% hatte er 'sich schon in Heidelberg einschreiben lassen. Seit dem 21. April 1548 bis Frühjahr 1550 sehen wir ihn in Tübingen, dann ein Jahr in Wien, 18 Monate in Ingolstadt. Wohl in Wien hatte er mit Thomas Pegäus ${ }^{1}$ ) Freundschaft

1) Veit Nuber, der von Wittenberg nach Österreich gegangen, am 6. Angust 1563 in Steyr eingetroffen war, meldet unter dem 20. Augast Panl Eber: „M. Thomas Pegaens, qui tibi autor fuit, huo 
geschlossen, vielleicht in der Folgezeit auch unter ihm an der Schule zu Stevr in Österreich anterrichtet. Am 14. September 1558 hatte er die Lencorea bezogen and war am 1. Mai 1560 in die Artistenfakultä aufgenommen wordeu. 1559 hatte er in Wittenberg erscheinen lassen: „Explicatio dilucida epistolae Judae" und "Historia de quodam episcopo a maribus consumpto". Melanchthon und Peacer waren vor anderen seine verehrten Lehrer, mit Jastas Jonas, dem Lehrer der Rechtswissenschaft, stand er in enger Verbindang. Dessen warme Empfehlung, die Vorliebe des Hohenzollern in Königsberg für seine stiddeutschen Landsleate, vor allem die Aussicht and Hoffnang, in Weigel einen gelehrten Richter in dem Osianderschen Streite zo erhalten, der vielleicht seinen verehrten and geliebten, seit 1550 in der theologischen Welt so verketzerten "Vater in Christo" wieder za Ehren brächte, bestimmte den Herzog Albrecht, ihn in seinen Dienst zu nehmen. Fast nmgehend, am 25. März 1561, lieB er ihm schreiben: ${ }^{1}$ ) „Nachdem wir in unserem Furstentum gater, christlicher, geschickter Pfarrherren und Seelsorger wohl benotdarft und derselben gern etliche in diesen Landen haben wollten, ihr aber ans everer Lehre, Geschicklichkeit, eneres Lebens and Wandels halben gerthmt seid worden, so hätten wir wohl gnädige Neigang, daß wir euch unter uns in einem Predigtamt wissen möchten, und wollen euch

mittendi concionstorem aliquem ante meum adventum, a senatu dimissionem petiit propter maltiplices persecutiones, quas patitur ob detestandam ingratitudinem civium strenue doctrinam evangelii et omnes bonos artes contemnentium. Utinam et ego aut huc renissem nanquam aut bona conscientia statim abire liceret ${ }^{4}$. Am 31 . Juli 1565 schrieb Eber an Pegäns: "Quod ad litteras tass attinet, peto mihi decepto veniam dari. In posterum ero fautior incidens in alienos, cum quibus non modicum salis absampsi, ne temere externae speciei et sono credam. Tuam amicitiam nt semper magnifeci, ita in posternm quoque officiis, quibns potero, tueri studebo." Am 29. Juni 1568, da der Steyrer Rektor ihn um einen Lehrer für seine Schule gebeten batte, meldet ihm Eber, daß er ihm Daniel Möller sende, der vier Jahre in Witter. berg studiert und jetzt im Sommer den Magistergrad erworben habe. Zagleich tröstet er ihn über den Ehebrach seiner Frau.

1) Dieser Brief ist wie sämtliche anderen Urkanden dem Steatsarchiv in Königsberg entnommen. 
daranf hiermit gnädigst rociert haben. Begehren mit Gnaden, dab ihr each ins Förderlichste allbero za uns in eineu Pfarrerdienst begeben wollet. Bei euerer Anknnft sind wir bereit, uns mit each des Unterhalts halbeu mit Guaden zu vergleichen. Im Falle ench hierin etwas Hinderliches vorfiele, so wollet solches gegenwärtigem Johann Funck anzeigen. Ihm haben wir Befehl gegeben, deshalb allenthalben mit each za reden."

Im April verhandelte Funck, der nach Wittenberg gekommen war, um den Theologen sein Glaubensbekenntnis vorzalegen, mit seinem Landsmann Weigel und bestimmte ihn, dem Rufe des Herzogs Folge zu leisten. Da er ein Nüroberger Stipendium genossen hatte und deshalb dieser Stadt verpflichtet war, versprach Funck, der nach Nurnberg reisen wollte, ihm dort Entlassung za erwirken. Während er nun nach Leipzig ${ }^{1}$ ) and Sijddeatschland aufbrach ${ }^{2}$, zog Weigel nach Königsberg. Am 2. Juni 1561 wurde er hier als "reverendus vir pietate et eraditione praestans" ins Album der Albertina eingetragen. Eine Ordination in Wittenberg muBte ihm nach Ansicht des Herzoge gröBerere Geltung und Antorität verleihen, deshalb ging er zugleich als herzoglicher Bote an Johann Major und Justus Jonas auf Anordnung Albrechts noch einmal nach der Elbstadt zarilek. Am

1) Am 31. Mai 1561 schrieb Pfeffinger ans Leipzig dem Herzog Albrecht über Funcks Konfession.

2) Nach seiner Rückkehr nach Königsberg schrieb Fanck am 15. Jnli 1561 an Paul Eber. Vergl. Codex Gothanus chart 123 BI. 354. ${ }_{n}$ Volui tibi ad rescribendam ansam praebere, donec ipse ad nos in Prussiam nostram, ut aliquoties T. H. percupere coram intellexi, bonis avibus venias. Quod at commodius citinsque fieret, dedi operam, ut ab ill. duce Alberto per literas vocarere, efficique simul, ut ad electorem itidem darentur literae, quibus ut D. T. huo venire atque ad unum mensem nobiscum esse licest familiariter petit. Quas autem ob causas petitio ista fiat, potest $D$. T. ex ill. principis mei literis, quas hisce meis adinnctas habes, una cum transsumpto ant descripto earum, quae sunt ad principem electorem, intelligere. Norit deus, quam bilari vultu animoque serenot pius princeps literas vestras acceperit, legerit, relegerit, quam avide etiam, quid inter nos sit actum, me referente ac declarante andierit. Saepins in haec verba eropit: ${ }_{n}$ Wolt got, das ich mein leben volend mit solchen lenten vnd mit solchen Disputationen möchte hinbringen". 
24. Juni traf er hier ein, händigte Major das fär ihn empfangene Geld und Schreiben ein, lieb sofort anch in der Schwenckschen Offizin seinen Tranergesang auf Melanchthons Heimgang dracken, den er anf der Reise niedergeschrieben oder vollendet hatte. Da er am 30. Jani an den Herzog schrieb'\}, konnte er seinem Briefe ein Exemplar seines Epicedinm beilegen ). Als er seinen Freunden seine Berufung nach Preußen augezeigt hatte, hatte er sie nm Gratulationen and Geleitgedichte gebeten. Er wollte sie drucken lassen, mit nach Krnigsberg nehmen und dort ausstrenen, um den Anschein eines allseitig geschätzten und hochverehrten Mannes za erwecken. Bis sie eintrafen, wartete er mit der Einholung der Ordination und aeiner Abreise.

Am 19. September(1561) starb in Wittenberg ein78 jähriger Pfarrer Michael Faust. Der Universitätsschrift, in der der Rektor die Studenten zur Teilnahme an dem Begräbnisse aufforderte, gab Weigel wie sein Landsmann Georg Mauritius, Jakob Alutarius ans Herborn, Georg Aperbach ans Erfurt and Joh. Reymann ans Löwenberg etliche Verse bei. ${ }^{8}$ ) Etliche Tage später veröffentlichte er die inধwischen eingegangenen Geleitsgedichte seiner Freaude.*) Einen Lobpreis auf die Lencorea fugte er ihnen bei. Am 5. Oktober ordinierte ihn Paul Eber.

1) $\nabla$ gl. Beilage I. Am 4. Juli 1561 schreibt Paul. Eber dem Berzog: „Cum voluptate audivi ex M. Weigelio et deo gratias ago, quod terribilis illa expeditio Johannis Basilii, magni dncis Moscoram, impedita sit". Im weiteren empfiehlt er den herzoglichen Alumnus Christian Farnhed, der lïnger als ein Jahr bei ihm gewohnt habe.

2) Epicedion in honorem et memoriam obitus reverendi et incomparabilis viri d. Philippi Melanthonis, patris et praeceptoris nostri summe colendi, scriptum a. M. Georgio Weigelio Noribergensi. Witebergae excudebat Laurentius Schwenk. 1561.

3) Epitaphium scriptum venerando et optimo seni D. Michaeli Fausto pie Wittebergae mortuo 19. Septembris 1561, cum esset egressus annum aetatis 78., a. M. Georgio Weigelio Noribergensi.

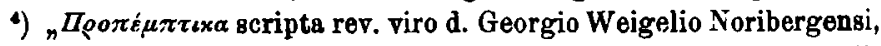
liberalium artium magistro, vocato ad ministerium verbi divini ab ill. Borussiae principe Alberto Seniore, Witeberga discedenti. Witebergae excudebaut haeredes Georgii Rhaw $1561^{\circ}$ in $4^{\circ}$, drei Bogen. Dreizehn Freunde, daranter Justus Jonas und Thomas Pegäus aus Landeshnt, Rektor in Steyr, bringen ibre Wünsche dar, zam Schluß „ad Witebergensem academiam $\mathbf{M}$. Georgius Weigelins." 
Weigel war Pbilippist and trat als solcher in dem gat latherischen Königsberg sofort mit aller Schärfe auf. Schon seiwe ersten Predigten erregten AnstoB, die, welche er am 21. Dezember 1561 in der SchloBkirche in Gegenwart der Herzogin, der Hofräte, des Prägidenten Johann Aurifaber bielt, fachte fast einen Aufruhr an. Er lengnete die Ubiquität Christi, seine Realpräsenz im Abendmahle; nur geistlicher Weise werde der Leib des Herrn genossen ${ }^{1}$ ). Fast einmtitig erklärten sich die Theologen wider ihn. Ihr Wortfuhrer warde Epplin, der ältere Hofpredigèr. Von Anfang an bestand zwischen diesem und Weigel ein gespanntes Verhältnis. Justus Jonas hatte seinen jungen Freand schon in Wittenberg gegen diesen älteren Theologen eingenommen \%). Als Epplin jetzt auf der Kanzel sich wider Weigel und seine Predigt rom vierten Adrentssonntage wandte, schrieb er ihm einen Brief ${ }^{*}$ ), der recht bezeichnend ist fur die Weise, in der er seinen Kampf fuhrte: "Ich kann mich nicht genugsam wandern der großen unverschämten Leichtfertigkeit, damit da die allergelehrtesten and heiligsten Leate anserer Zeit ganz liederlich bei dem gemeinen Volke angiebst and mit unerhörten Schmähworten besohwerst. Es pflegen sonst, die anf andere Lugen dichten, Diebe za sein, du hast hente auf deine Präzeptores gat and böslich gesprochen (o da unverschämtes Maal, das allein Schmach und Schande ausspeien vermag), dab sie mit diesen Worten, ihre Hände seien voll Blat, die Worte des Abendmahls, das ist mein Leib' auslegen. Ich habe niemals anderen, die dir grobe, anverschämte Leichtfertigkeit zugemessen, glauben wollen; nuu aber ma $B$ ichs glauben, daB da ein hoffärtiger, theraus unver-

1) Mathias Wanckel schreibt unter dem 18. März 1562 aus Kemberg:

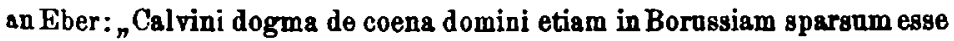
ex corde doleo et certe nescio, qua conscientia vestras sententias et formulas loquendi de coena sacrosanctissima ecclesiae laboranti non committatis".

2) Am 13. April 1559 in einem Briefe aus Leipzig hatte er gemeint anch den Herzog vor Epplin „und seinen Narrenbüchern" warnen zu müssen.

3) Löscher, Histeria motuam II, 218 ff. Im Einzelnen anterrichten tuber den Streit zwischen Weigel und Epplin zwei Aktenstücke vom 10. 3lärz und 11. April 1562 im Königsberger Staatsarchive. 
schämter, heilloser, unwissender Mensch seist ... Ich will es mit Gott bezeugen, dab ich heate nichts denn eine eselische und cyklopische Unwissenheit gehört habe. Wabrhaftig ich habe einen Esel gesehen auf der Kanzel in einer leinenen Hant rerkleidet." Und so geht es fort, jeder Satz eine schwere Kränkang and Beleidigang. Ein Glaubensbekenntnis, das Weigel weiter heransgab und in dem er mit aller Entschiedenheit die symbolische Dentung des Abendmahls vertrat 1), begegnete naturlich dem schroffisten Widersprach.

Bald stritt man nicht nor in Königsberg wider ihn und suchte dem 73 jäbrigen Herzoge seine Entlassang abzadrängen. Wie im Osianderschen Streite die Kande von alle»s Vorgängen in Königsberg sofort nach Wilna flog ${ }^{2}$ ), wie Osianders Gegner am königlichen Hofe wider ihn and seine Anbainger Stimmang machten ${ }^{3}$ ), so denunzierte man jetzt beim polnischen Herrscher Sigismand August anch Weigel als einen Unrahstifter. Der König sah sich bewogen, an den Herzog za schreiben and ibn eindringlich vor dem Zwietrachtprediger za warnen. Als Albrecht antwortete, dab

1) „Panis est corpus Christi non proprie vel realiter, sed improprie, figorate vel sacramentaliter. Mandncare corpus Christi et bibere sanguinem eins est in Christo manere et Christum manentem in se habere". Löscher II, 217.

2) Vgl. Pohibels Brief aus Wilna rom 4. Okt. 1ij1. Möller. Andreas Osiander S. 459. Schon am 29. Sept. hatte er ans Wilna dem Herzog gescburieben: Man hat hier auch der prädicanten zu Konigsperg, e. f. g. anch hyn vnd her gedacht, der eyne besser dan der ander. Sprechen, der hertzog sambt der hertzogin haben eyn sonderliche lere, das frewlein anch besonder, das hoffigesindt ind dy rethe dergleychen. In den stoten geht es auch seitzam zu ... Es wero besser gewesen, das dy predicanten den predigtstuel eyn zeytlangk, damit es voder dy gemeyne nicht kommen were, gemyden, bis s. sich vereyniget vnd verglichen hetten. In Summa es begeben sich mancherlei worthe vnd rede, welche ich jtzo jn der feder mus rowen vod bleibten lassen, jedoch bleibt solchs e. f. g. zn gelegener zeit vaverhalten."

8) Krakan, den 22. März 1553 schreibt Pohibel dem Herzog: "Ich kann e. f. d. nit bergen, das in kortzen tagen der her marienborgische woywoda durch eignen pothe an meinen hern [den königlichen Vorschneider Gabriel Tarlo] geschrieben rnd jm angezeigt, wie das eyn seltzam tamult vnd zwyspalt za Konigsperg des predicanten halbeu d. Morlens erhoben, alzo, wo dem nicht vorgekommen, eyn 
er anrahige Geister in seinem Herzogtnm nicht dalde, gab er am 1. Mai seiner Freade dariber Ausdruck, unterlieB aber nicht, von nenem za warnen ${ }^{1}$ ).

Den Hofmeister der Königin, Erhard von Kunheim, hatte der Herzog im Verdachte, den König wider Weigel eingenommen za haben. Aber dieser Brader des Schwiegersohnes unseres Lather war wohl anschuldig. "Die ganze Stadt allbier ist des Redens voll gewesen, und man hat sich mit Briefen umbergetragen, so von E. F. D. Hof anher gescbrieben auch von denen, so E. F. D. nicht wenig vertranet“, schreibt er Wilna, den 3. Mai 1562 za seiner Rechtfertigung. Um sich noch mündlich za verteidigen, kam er im Juni nach Königøberg ${ }^{2}$ ).

gros blutvorgyssen hyraus entstanden were. Jdoch hinge es noch an der wage n8w., mit andern vil mehr vmstendigen worthen, wie dan seyner gnaden eigen hantschreiben mitbringt, meynem herm zn erkennen geben rnd ferner gebeten, das meyn her bey der kön. majt treulich sollicitiren wolde, damit jre majt eyn eigen potschafft an e. f. g. abfertigen thete, damit e. $f$. g. vermanet, von solchem vornehmen der zwyspäligen lehren abzustehen etc. Were sonst zn besorgen, eyn schrecklichers hyrans zu erfolgen. Sind sonst auch zwei briefe bey meynes herrn briffe gewest an die kön. majt, eyner von dem herrn bischoff von Ermelant, der ander von gedachtem herrn woywoden. In dem rad vff solch schreiben hat mich meyn herr gefragt, was er jn dem fall thun solde, ob er solchs jrer majt anzeigen solde oder nicht, weyl er sonst von nymandt schreiben hette. In dem alzo geschlossen, das sich mein her mit worthen vad dergleichen nix kegen ire majt einlassen solde". Im weiteren bittet er den Herzog um einen genauen Bericht, ndamit, ob hernachmals sich ethwas an tag geben würde, dem mit der warheit desto bas vorzukommen". Vgl. schlieblich auch Pohibels Brief rom 24. April 1553: ${ }_{n}$ Was den d. Morlein belangt, - hat man alhir vil seltzam+r redenn vad sonderlich an dem, das etzlich vill frawen ind juntfrawen gleich eyner procession jns schlos zu Konigsperg gangen vad e.f. g. durch dy fraw Venedigeryn eine supplication zustellen wollen, welche e. f. g, nicht angenommen, sonder sie sembtlich in jre behausunge abzugehen befelich geben, das alzo mit eynem gesange eynes psalmes gescheen. Wirt aber wenig hyvon am hofe gedacht, anßgenommen dy aus Preußen hier ankommen, dy haben vil geschwetz, mehr andern leuten zu gefallen, denn der warbeit enlich"

1) Vgl. Wotschke, Abrahsm Kulvensis. Urizunden zur Reformationsgeschichte Lithanens. Altpr. Monatsschrift XLII S. $237 \mathrm{f}$.

2) Über diesen Kunheim vgl. Wotschke, Wittenberger Berichte ans der Interimszeit. Jahrbuch für Kirchengeschichte der Provinz 
Der altersschwache Herzog and die wenigen Reformierten in seiner Umgebang, Friedrich von Kanitz ${ }^{1}$ ), Friedrich von Aulack $^{2}$ ) and Schwerin, konnten Weigel gegen den allgemeinen Unwillen nicht schutzen. Er ging deshalb, als ihm in Königsberg die Kanzel verboten warde, nach Lithanen, um dort Unterstutzang za sachen, anch die Gunst des Fursten Radziwill fur sich za gewinnen. Die dentsche latberische Gemeinde in Wilna, welche erst im November 1560 in M. Simon Wanrab einen Pfarrer, Anfang 1562 in einem Konigsberger Kaplan einen zweiten Prediger erhalten batte, lehnte ihn ab, aber bei den lithanischen reformierten Pastoren fand er freandliche Aufnahme. Mit den Zurichern nod Genfern waren sie im vergangenen Jahre in Verbindung getreten, erst am 23. Januar war einer unter ihnen, Martin Czechowicz. von einer Gesandtschaftsreise nach Gent ${ }^{8}$ ) heimgekehrt, jetzt dachten sie darch den Wittenberger Magister auch Beziehnngen za den Pbilippisten anzaknupfen. Durch sie fand er Eingang am Hofe des Fürsten Radziwill. Hier lernte er den theologisierenden Arzt Blandrata kennen, der im nächsteı Jahre nach Siebenblirgen ging, vor allem aber den Geheimschreiber Johann Macrinski, einst Pellikans Hansgenosse in Ztrich, in Wittenberg Melanchthons Schuler. Er warde ibm

Sachsen 1913 S. 5 ff. Knnheim folgte der Kðnigin Katharina Herbst 1566 nacb Österreich. Linz, den 23. April 1571 empfiehlt die Königin Volmar von Kanheim aus Preaßen dem Kurfürst August von Sachsen. Vor fünf Jabren sei ihr dieser Kunhrim als Edelknabe zugesandt, jetzt möge ihn der Kurftirst in seine Diensto nehmen.

1) Dieser Kanitz war neben dew Abenteverer Skalich ond dem Radziwillschen Sekretär Maczinski der Testamentsvollstrecker Lismaninos. Vgl. Wotschke, Francesco Lismanino, Zeitschrift der Hist. Gesellschaft Posens 1903 S. 323 ff. Uber die heimliche Ehe, die er in Konigsberg geschlossen, vgl. Wotschke, Wittenberger Berichte S. 7. In Breslan war der reformierte Craco sein Freund. Anf dessen Empfehlung snchte er 1561 den kalvinisch gerichteten Birkenhan in Preußen zu versorgen. Gillet I 241.

3) Über Aulack vgl. Wotschke, Herzog Albrecht und Graf Raphael von Lissa. Altpr. Monatsschrift XLVI S. 484 ff. and 489.

7) Das Schreiben vom 9. Oktober 1561, das er von Calvins Hand mitbrachte, bewahrt noch hente das Archiv der reformierten Gemeinde Wilnas als kostbare Reliquie. Die Monumenta reformationis Polonicae ot Lithusnicae bieten es im Gleichdruck an erster Stelle, 
ein Freand and schrieb für sein grobes polniseh-lateinisches Lexikon, das jetzt nach jahrelangen Bemtihangen endlich tn Königsberg gedrackt werden sollte, aber erst 1564 wirklich erschien ${ }^{1}$ ), ein empfeblendes Gedicht an den Leser Furst Radziwill wandte ihm seine volle Gunst zu und war geneigt, ihn in seine Dienste zn nehmen als Erzieher seines ältesten Sohnes, des 13 jährigen Nikolans Christoph '), dem Vergerio 1556 die treftliche Kinderlehre "Lac spirituale" des evangelischen Spaniers Juan de Valdes gewidmet batte ${ }^{3}$ ), ihn mit diesem anch nach dem Anslande, nach Deutschland, der Schweiz and Italien zu senden. Wilna, den 26. Jani (1562) schrieb er daron dem Herzoge and bat, Weigel ans seinen Diensten zu entlassen $\left.{ }^{4}\right)$.

Albrecht hatte unterdessen alles versucht, den durch Weigel erregten Sakramentsstreit za dämpfen, dabei anter dem Einfluß Johann Aurifabers anf Wege gesonnen, therhaupt 2 wischen Latheranern and Reformierten einen Ausgleich za schaffen. Seine Theologen mabten ihm im Mai Gutachten ausarbeiten ${ }^{5}$. . Obwobl mit Ausnahme Aurifabers sie alle kein Vertranen za einer Unionsaktion hatten, hielt der zähe Herzog doch an ihr fest. Im Jali mabte der aus Lithanen heimgekehrte Weigel eine Reise nach Súd-

1) „Lexicon Latino-Polonicam ex optimis latinae linguae scriptoribus concinnatum".

2) Er war am 2. Angust 1549 geboren.

3) Vgl. Wotschke, Eustschius Trepka. Zeitschrift dor Hist. Gesellschait der Prov. Posen 1903. Wie dem siebenjährigen Knaben die lac spirituale widmete Vergerio dem 16 jährigen Jüngling, als er hei inm (1565) in Tübingen weilte, seine Risposta in quartro libri divisa ad una invettiva di fra Ippolito Chizzuola. Unter dem 31. März dieses Jahres hatte ihm Lorenz Tuppius in Strabburg schon zuge8chrieben: Adversus synodi Tridentinas restitationem opposita gravamina. Vom 15. Mårz d. J. ist datiert Sturms epistola de refutatione Tridentini concilii an Radziwill den Vater.

4) Albrechts Antwortschreiben vom 12. Angust 1562 bei Wotschke, Culvensis S. 241.

6) Vom 19. Mai 1562 sind die Gutachten Funcks, Epplins, Anrifabers, Vogels datiert, vom 22. Mai das Jagentenffels, vom 6. Oktober das des Sickius. Unter dem 21. Oktober warnt Funck den Herzog vor der kalvinischen Abendmahlslahre. Vgl, anch Hase, Herzog $A_{\text {lbrecht }}$ und seine Hofprediger S. 280. 
deatschland antreten, um mit den Heidelberger and Wurttemberger Theologen zu verhandeln. Im Augnst sehen wir ihn in der Neckarstadt, wo er Olevian and Ursin sein Bekenntnis vorlegte. Nattrlich fand es deren Zustimmang ${ }^{1}$ ). Dem Herzog schrieben sie, daß es biblisch wohl begrtundet sei. Weigel rahmten sie als einen gelehrten und beherzten $\mathrm{Mann}^{2}{ }^{2}$ ). Daftur begegnete es in Stuttgart, wohin Weigel sich nun wandte, um so entschiedenerem Widersprach. Brenz and Andreä wiesen es mit rollem Nachdrack ab. $\mathrm{Da}$ Weigel bei ihm verharrte, wurde der antberbrtickbare Gegensatz offenbar. Vergerio in Tubingen, zu dem er im Anftrage des Herzogs im Dezember kam \%), hielt mit seiner Meinong vorsichtig zuruck. Er wollte nirgends anstoben. Im Jannar ging Weigel auf eine Forderung Brenz' za neden Verbandlungen nach Stuttgart *), aber der Zwiespalt warde durch diese weitere Aussprache nicht geringer. Selbst Vergerio, der in seinem Schreiben an den Herzog vom 23. Jannar 1563 der gastfreundlichen Anfnahme gedenkt, die anch Weigel ruhmen musse, sieht sich jetzt veranlaBt hinzozufugen: „Reliqua in homine non laudo“.

Manchen Bekannten aus seiner Stadienzeit 1548-1550 hatte Weigel in Tubingen wieder getroffen, zo den hier seit dem 14. Angust $15 € 0$ stadierenden Lithauern ${ }^{5}$ ) Beziehangen angekntipit. Für seine Abendmablslebre konnte er letztere indessen nicht gewinnen. Ihr Präzeptor Georg Zablocki, seit seinem Stadinm an der Leacorea 1540 ein uberzengter Latheraner ${ }^{6}$ ), der fulr seinen Glanben in der Heimat 1544 auch gelitten hatte, wies ibn ab. Anfang Febroar wollte Weigel heimreisen. Schon hatte er am 3. zwei Wagenpferde

1) Vgl. Ursins Brief an Crato. Neue Heidelberger Jahrbiicher XIV, S. 60 und Gillet, Crato von Krafftheim I S. 264.

2) Vgl. Oliviang Schreiben an Calvin vom 3. April 1569 U.C. XIX Nr. 3925.

3) Vgl. Vergerios Brief an den Herzog Christoph vom 10.Januar 1563 Schott und Kansler, Vergerios Briefwechsel S. 369.

) A in 11. Nov. 156ะ bat der Pole Valentin Maslorius (die Tübinger Matrikel liest Marlenins), seit dem 16. Sept. 1561 in Wittenberg, die Tübinger Hochschule bezogen, am 25. Januar 1563 der Preuße Joh. Hermann. IhrTübinger Studium hängtwohl mit Weigels Reise zusammen.

b) Vgl. Wotschke, Culvensis S. 212.

") Homo don indoctus et exinie Brentianus" nennt ihn Bnllinger in seinem Brief rom 31. Mai 1563 an Beza. O. C. XX Nr. 3959. 
gekauft, schon schrieb an 6. auch Vergerio den Brief, den er ihm für den Herzog mitgeben wollte, da scheint das Ausbleiben der Briefe des Brenz und Audreae für den Berzog ibn zo weiterem Bleiben bestimmt zo haben. Ja am 20 . Febraar ging er, von Vergerio an Ballinger emplohlen, noch nach Zürich ${ }^{1}$ ). Naturlich war er hier wie in Heidelberg den Theologen hochwillkommen ${ }^{2}$. Diese stimmten ihm za, Bullinger entlie $b$ inn mit eivem warmen Brief an den Herzog und seine Räte. Die hohen Worte, mit denen er nach seizer Rückkehr in Tübiugen die in Zưrich gefundene Aufnahmo ruhmte, bestimmten auch drei der jungen lithauischen Barone mit ihrem Lehrer Zablocki, Bullinger aufzusuchen. Vergebens suchte der Zuricher Theologe sie fur seine Abendmahlslebre zu gewinneu. So gewandt vertrat Zablocki ihm gegentber den latherischen Standpankt, daß Ballinger meinte seine Geufer Frennde, die er mit seinen Schtlern gleichfalls anfsuchen wollte, vor ihm waruen za mussen. „Pradentes este ${ }^{48}$ ). Im März, flinf Monate bevor der ihm im vergangenen Jạhre zugedachte Schtuler Nikolaus Christoph Radziwill, der spätere Jerasalemfahrer, nach Stuttgart $\operatorname{kam}^{4}$ ), trat Weigel die Heimreise nach Königsberg an.

Noch ein Brief des Herzogs Albrecht, der im März in Tubingen eintraf, empfahl Weigel dem Vergerio, aber andere Schreiben aus Preußen meldeten, da 6 Weigel angesichts des allgemeiuen Widerspraches schwerlich in Königsberg werde bleiben können ${ }^{5}$ ). Albrecht selbst warde darch eineu Brief

1) Wotschke, Briefwechsel der Schweizer mit den Polen S. 166.

2) Ballinger in seinem Tagebuch unter 1563: D. Georgins Weigelius, concionator principis Prusseni, venit hac mense Februario Sturgardia, nbi contulerat cum Brentio. Contulit et mecum de coena et consensit. Tulit a me literas ad principem eiusque consiliarios". Vergl. auch seinen Brief an Calvin vom 20. April 1563. $0 \cdot \mathrm{C}$. XIX Nr. 3937.

3) In den Briefen rom 31. Mai 1563, O. C. XX Yr. 3959 und 3960

4) Am 3. Aug. 1563 traf Badziwill in Stattgart ein, am 9. August schrieb Herzog Christoph für ihn nach Straßburg. Von dort im Augast 1564 darch die Pest vertrieben, kam er nach Tübingen und studierte hier bis zum August 1566. Am 4. Sept. 1566 sehen wir ihn bei Bullinger.

5) Vgl. Vergerios Schreiben an Herzog Christoph vom 31. März 1563. Schott and Kaulser S. 381 . 
Herzog Christophs von W trttemberg bedenklich $^{1}$ ). So vertrøstete er Weigel, als dieser Bericht erstattete, anf späteren Bescheid, gab solchen aber nicht, obwohl Weigel darnm anhielt. Daftur forderten die Räte in Abwesenheit des Herzogs, als dieser im Joli in Kanen beim Könige weilte, ron ihm Amtsniederlegung, and der Burggrat lieB ihm , fein spottisch Dienst and Tisch absagen " ). Obwohl ihm eine nehrliche Heirat in Königsberg angetragen war, verlieb er jetzt Preaben and ging nach Lithanen. Hier hoffte er als Prediger Versorgang zo finden, hier batte er dorch die lithanischen Stadenten in Tubingen neae. Frende gewonnen. Anf Grand der Briefe die er tuberbrachte, and der Berichte, die er erstattete, mag Wilna, den 26. September (1563) der Marscball Eustachins Wollowicz, seinen Neffen von der suddentschen Hochschule abgerufen habed. Am 17. November bittet Weigel den Herzog um Entschaldigung, daB er ohne formelle Entlassung nach Wilna gezogen sei ${ }^{8}$ ).

Radziwills jügere Söhne Georg, Stanislans und Albert waren erst acht, sieben und ein Jabr alt, sie brauchten zar Zeit noch keinen Gelehrten zum Erzieher. Erst Herbst 1566 warde fur sie ein des Lateinischen, Dentschen und Polnischen kundiger Student gesucht ${ }^{4}$ ), der die jungen Fursten nach

2) Vgl. Vergerios Brief vom 8. Sept. 1563 a. a. O. S. 394.

3) "Audimus, quod princeps suos theologos et nobiles in potestate non habeat" schrieb Ursin.

7) Vgl. Wotschke, Culvensis S. $242 \mathrm{ft}$.

4) Der Wilnaer Stadtrogt Augustin Rotundus schreibt Grodno, den 3. Angust 1567 an Hosius: „De palatini Filnensis filio ex Itntia reverso constans hic fama est, ipse enim eum nondum vidi, catholicum esse factum. Quin et quae d. Mielieczki, palatini Podoliae filio, nupsit, eiusdem d. palatini Viluensis maxima nata flis catholica esse facta dicitur in mariti catholici ex Luthes ano facti gratian. Tres quoque reliquae natu minores in domo insignis matronae $d$. Voinicensis educantur et eandem, quam domina Voinicensis profitetur; catholicam doctrinam profitentur." Am folgenden 13. Septembermelıet eraus Wilua: -Filii palatini Nesuesi sub praeceptoribus et paedagogis'haereticis educantur, sed spes est eos quoque ad catholicam ecclesiam redituros tratris sororumque exemplo.“ Doch hat Nikolaus Mielecki ( $†$ o. Februar 1585) orst viel später seinen evangelischen Glauben abgeschworen. Wohl hatte ihn schon 1569 Skarga zu bekehren gesucht, aber erst mehrere 
Leipzig begleiten konnte. ${ }^{1}$ ) Seit Wintersemester 1570 sehen wir sie dann dort, wo der bekannte Arzt Simon Simonius ans Lucea ibre Stadien leitete ${ }^{2}$ ). Der Furst batte ftr Weigel kein Amt, vielleicht trug dieser auch nach dem, was er in Zurich tber Blandrata and dessen Gönner Radziwill gehört batte, Bedenken in seine Dienste zo treten. Tatsächlich hatten ja die Tritheisten den gröBten Einflub an Radziwill gewonnen, and nur der Tod bewabrte ihu davon, entschiedener Antitrinitarier zu werden.

Da nahm ein anderer lithauischer Magnat Weigel in seine Dienste, Jobann Chodkiewicz, der Hauptmann ron Samogitien. Schon als Kind war dieser in Elternhanse evangelisch erzogen worden, als Stadent hatte er 1547 in Königsberg, seit 1549 in Leipzig evangelisches Gemeindeleben kennen gelernt, vortbergehend anch in Wittenberg dessen Matrikel seinen Namen allerdings nicht bietet, zusammen mit Stanislaus Warsehewicki, dem Sobne des

Jahre später gelang es Benedikt Herbst. Seine Gattin Elisabeth Radzi will, die große Bibelkennerin, die sich den Antitrinitariern angeschlossen batte, kehrto 1593 in den Schoß der römischen Kirche zarück.

1) Am 29. Oktober 1566 bat Damian Nicossowius, don jungen Radxiwill nach Deutschland begleiten xu dürfen. Fr erhalt den Bescheid: nF. D. wollen zufrieden sein, daB er mit des Radziwills Sohne hinauszieho nnd seine studia proseqnire, doch dab or mit des Rektors Vorwiesen abscheide and ihrer fürstl. Durchlancht oder nachkommender Herrschaft hernach vor anderen Herren diene, sich auch deshalb obligiere“. Seine Verpflichtnng bei Wotschkes Vergerios zweite Reise S. 317. Am 7. Joli 1567 stellte anch Matthäns Motzarus, der den 6. Mărz 1567 noch um ein Stipendium gebeten hatte und gleichfalls mit jungen Lithauern nsch Deutschland gehen wollte, einen Revers aus; nach irei Jahren nach Preaßen zurtickzukehren, doch vgl. Beilage III.

7) 1572 kehrten die Brüder nach Lithanen earück ond wurden hier ron Skarga, damals Rektor des Wilnaer Jesuitenkollegiums, für den Katholizismus gewonnen, nachdem ihr ältester Bruder schon $\mathbf{1 5 6 7}$ in Italien übergetreten war. Georg wurde 1581 nach Ruckkehr von einer Reise nach Italien Bischof von Wilna, dann Kardinal and Bischof von Krskau ( +21 . Jan. 1600 in Rom), Stanislaus richteto den MeBgottesdienst in Olika wieder anf. Albrecht starb am 13 . Juli 1592 als Marschall von Lithauen, zwei Monate nachdem er die Braut des Königg von Österreich nach Krakan geleitet hatte. Skarga macht den Arianer Cikowski filr seinen Tod, der ob potionem quandam alchimisticam erfolgto verantwortlich. 
Warschaner Kastellans Johann Warschewicki, dem späteren Jesuiten '), auch za Melanchthous FuBen gesessen 2). Sein Vater, der Kastellan von Troki Hieronymus Chodkiewicz, war ein Gönner des Staphylus und hatte diesem gelegentlich seiner lithauischen Reise April 1549 manche Förderang erwiesen. 1556 empfahl ihm der Herzog Albrecht einen Prädikanten Matthias Virowitta, der in Königsberg stadiert hatte and in Samogitien ein geistliches Amt suchte. Auch Gregor Chodkiewicz, das andere Hant dieser hervorragenden lithauischen Magnatenfámilie, der Wilnaer Kastellan und Oberfeldberr († 1572), war ein Frennd der Reformation, die er in seiner Jogend am Hofe Herzog Albrechts kennen and lieben gelernt batte. Seinen Söhnen Andreas (geb. 1549) and Alexander (geb. 1550) gab er in Johann Mylias ans Liebenroda in Thturingen, dem namhaften Dichter der lateinischen Renaissancepoesie, dem Übersetzer von Lathers kleinem Katechismus ins Lateinische und Griechische and späteren Professor der hebräischen Sprache in Jena ( $\uparrow$ 3. Juli 1575), einen evangelischen Erzieher. An den Königsberger Hof schickte er sie, daB sie dort unter den Edelknaben des Erbherzogs "Zucht und alle Togend" lernten, später aber anch an den kaiser-

1) Geb. 1527. In Rom trat or 1567 zusammen mit Aquaviva in den Jesuitenorden ein, später arbeitete er in Rom, dann leitete er viele Ja'bre das Wilnaer und Lubliner Colleginm und diente sechs Jahro der Königin Katbarina von Schweden, der Jagellonin, als Beichtvater. Er starb am 3. Oktober 1591 in Krakan. Seine Übersetzung des Heliodor, welche 1555 in Antwerpen erschienen ist, ist datiert „Ex Warachewicze paterno rure 12. Cal. Aug. 1551".

2) Rostowski schreibt von Chodkiewicz in seiner Geschichto Lithanens: "Huic Varschevickins non modo notus erat, sed praecipua etiam familiaritate coniunctus ex eo iam tempore, quo ambo iuvenes nlim Vitembergae famoso literaram et haeresis suae magistro Philippo Melanchthone aci essent. ${ }^{*}$ Der Neulateiner Johann Mylins sagt in seiner Elegie ad magnificum d. Joannem Chodciewitium, Samogitiae praesidem:

„Ut Linus Herculeum mollivit pectus in arte, Posset ut humano commodus esse gregi,

Sic te Pierio madefecit fonte Melanchthon Cui similem nondum Teutonis ora tulit.

Illius e labris suxisti dogmata certa, Regula quae vitae sancta fuere tibi“. 
lichen Hof nach Wien. Hier ging ibnen verloren, was sie an evangelischer Erkenntnis besaBen.

Eine Hauptanfgabe Weigels war, dem in Lithanen am sich greifenden Antitrinitarismus entgegenzuwirken. Selbst sein Freund Maczynski war zo ihm ubergegangen ${ }^{1}$ ). Leider flieben die Nachrichten sehr spärlich, daB wir ron Weigels Arbeit in den nächsten Jahren, von seinem Leben überhaupt fast nichts wissen. Hat er den Fuhrern der lithanisehen Antitrinitarier, einem Gonesins, Czechowicz, Maczynski, Budny, Kryszkowski sich entgegen geworfen, dem Superintendenten Simon Zasins, dem Prediger Wedrogowski zar Seite gestanden? Hat er Bezas dogmatisches Sendschreiben vom 19. März 1565 das sehnstlehtig erwartet im Laufe des Sommers in Lithanen endlich eintraf ${ }^{2}$ ), wider die Gegner ansgespielt? Hat er mit Lismanino verhandelt, den wir 1564 and 1565 von Königsberg nach Lithauen reisen, Herbst 1565 gerade anch bei dem Marschall Georg Chodkiewicz sehen ${ }^{8}$ ). Hat Weigel 1564 die Ehe seines Herrn mit Christiane, der Tochter des trea evangelischen Krakauer Wojewoden Martin Zborowski, der

3) Wilna, den 13. Sept. 1567 schreibt Rotandus an Hosins: ${ }_{\text {Credo }}$ R. D. V. multo melins nobis scire, quam variis et horrendis sectis conspirent in Polonis haeretici, quae etiam ipsis haereticis non probantur, uti ex hoc literarum cainsdam J. Maczynoki, prioris palatini Vilnensis scriba, qui ante sacramentarius fuit, nunc et trinitarias et anabaptista esse factus dicitur, exomplo ad Pazum episcopum, si dis placet, Kijoviensem scriptarum cognoscet. Vidi ego es legi Grodnae typis excasos Polonicos libellos, quibus magis blasphemum in dei filinm Jesum Christom diei ant cogitari nihil potest ac ne dictnm quidem ant cogitatum unquan ab ullis haereticis existimo, de quibus fortasse Maczyngki in his litteris innnit. Tollitar enim in illis omnis omnium magistratuam antoritas, probatur libertas christiana, et rerum omnium communio instituitur, ordinum in ecclesia atqne adeo in repnblica omne discrimen tollitur, ne ullam sit inter regem et populam, principes et subiectos, nobiles et plebeios". Das Herrnhuter Archiv besitst eip Schreiben Maczynskis vom 28. Febr. 1558 ans Wiles an den PfarrerJohann in Stawischin, in dem er sich günstig über dieböhmischen Brüder susspricht.

2) Am 25. Hai 1565 befand es sich noch in den Händen des Radziwillschen Reisemarschalls Balthasar Lehwald in Tubingen. Am 28. April 1550 hatte dieser Radziwillsche Beamte einst die Lencorea bezogen.

7 Vgl. Wotschke, Francesco Lismanino. Zeitsch. d. hist. Gesellschaft d. Prov. Posen 1903 S, 307. 
jugendlichen Witwe oder nachgelassenen Brant des abentenernden Melanchthon- and Laskifreundes Jakob Heraklid Basilikus ${ }^{1}$ ) eingesegnet, getauft sein Söhnlein Johann Kari, den späteren Kriegsmann nnd gewaltigen Feldherrn, dessen Siege ther die Schweden bei Dorpat, Weißenstein uod Kirchholm ganz Europa anfmerken lieBen?

Der Kampf gegen die Antitrinitarier fubrte ihn mit dem großen reformierten Kämpfer Lithauens Andreas Volan zasammen, der 1531 in Neustadt bei Pinne (Provinz Posen) geboren war, 1544-1546 die Universität Frankfurt besucht batte, darauf seinem Verwandten Hieronymus Quilecki nach Lithanen gefolgt war, seit dem 5. Oktober 1550 noch etwa drei Jahre in Königsberg studiert hatte und schlieblich in Radziwills Dienste getreten war, nach dessen Tode 1565 anf seinem Landgate Bijuciszki bei Wilna lebte, soweit er nicht durch diplomatische Geschäfte in Anspruch genommen war. Vor allem aber trat Weigel Nikolaus Paz näher, der seit 1555 Bischof von Kijew war. ${ }^{2}$ ) Er gewann ihn, den einzigen Bischof in Polen, der wirklich den Übertritt zur evangelischen Kirche vollzog, ${ }^{3}$ ) fur die Reformation ${ }^{4}$ ) and bestimmte ihn, gegen die Tritheisten das altkirchliche Dogma in einer veuen Schrift za verteidigen. Vom 22. Juli 1566 vom Generalkonvent in Brest ist sie, die orthodoxa fidei confessio de una eademque dei patris, filii et spiritus sancti divinitate ac tribus personis, datiert. Ihr Verfasser gab ihr einen Brief ${ }^{5}$ ) Volans vom 1. April 1565 uber die drei Personen in Gott and die eine göttliche Essenz bei, Weigel eine empfehlende Beurteilang; ein Epigramm an den Leser und fromme Verse. Anfang Oktober 1566 sandte ibn Chodkiewicz von Kauen nach Königsberg, um dort durch die Daubmannsche Drackerei

1) Vgl. Wotschke, Joh. Laski und der Abenteurer Heraklid Basilikus. Archiv XVII S. 57.

2) $1583-1585$, wo or starb, war erKastellan von Smolensk.

7) Die Bisohöfe von Kamieniecz, Leslan nnd Samogitien sympathisierten wohl mit der Reformation, mochten aber das Opfer eines Ubertritts nicht bringen.

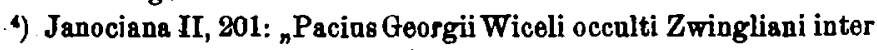
Polonise ac Lithuaniae proceres annis superioribus versati maxime artibus irretitus uxorem ducit."

5) „Datum in praediolo meo Bintiscano." 
das Schriftchen zu reröffentlichen. Ein Empfehlangsschreiben des Chodkiewicz an den Herzog, bei dem dessen Schwager Petras Zborowski im vergangenen Augast unter den polnischen Kommissaren erschienen war, förderte ihn, im November konnte er die Ruckreise antreteu. ${ }^{2}$ )

In den folgenden Jahren mag Weigel vielfach mit Friedrich Holsten aus Bunzlan zusammen gelebt haben. Dieser hatte 1565 das Präzeptoramt bei dem in Leipzig stadierenden Konstantin Chodkiewicz') angenommen nud war seinem Schtler nach Lithanen grfolgt. Erst 1569 kehrte er von dort nach Wittenberg zuruck, um in den Jahren 1572 1579 als Lehrer in den Brtdergemeinden zu Koschminek and Lissa noch einmal dem sarmatischen Osten za dienen. ${ }^{3}$ )

Mit steigendem Befremden sah Chodkiewicz anf die seit 1563 schnell wachsende kirchliche Zerrtttang seines Landes. Zu den griechischen and römischen Katholiken waren Latheraner und Reformierte getreten, ferner Tritheisten, welche die altkirchliche Trinitätslehre festhalten wollten ohne deren angeblichen Sabellianismus, Dystheisten, welche die Persönlichkeit des beiligen Geistes lengneten, Unitarier, Anabaptisten. Welche Spaltung zeigte allein seine nächste Verwandtschaft! Von seinen Schwagern waren Johann v. Kurzbach4) nnd Johann Zborowski gate Latheraner, Peter Zborowski damals noch ein Groner and Scbutzfreand des Stancaro, ${ }^{6}$ ) der eine eigene Sekte gegrtindet hatte and gerade 1565 mit besonderem Nachdruck ftr sie warb, Andreas Zborowski seit seinem Wiener Aufenthalte, Sommer 1560, $)$ strenger Katholik. Samuel, der am 26. Mai 1584 in Krakan das Blntgerdist

2) Vergl. Wotschke, Kulvensis S. 250.

?) Dieser Sohn des Georg Ch., des lithanischen Vorschneiders und Hanptmanns ron Bielsk, studierte seit 1562 in Leipzig. 1563 hat ihm L. Camerarins seine Praecepta vitae gewidmet.

3) Vergl. Wotschke, Graf Andreas von Lissa, S. 31.

4) Gatte der Anna Zborowska.

5) Vergl. Wotschke, Francesco Stancaro, S. 48. Peter Zborowski nod seinem Bruder Samuel widmete später der reformierte Saperintendent Paul Gilowski seine Katechismasanslegung.

-) Wintersemester 1557-1558 sehen wir ihn mit seinen Brüdern Samnel, Martin und Petrus in Frankfurt, seit dem 20. Januar 1561 in Wittenberg. Als er 574 seine Hochzeit feierte, war König Heiorich sein Gast. 
besteigen maBte, and Christoph Zborowski ${ }^{1}$ ) reformiert. doch bewarb sich letzterer 1567 am die Hand der griechischkatholischen Schwester des Wojewoden Bogdan ron der Moldan; seine Schwägerin Elisabeth heiratete allerdings erst September 1574 den bekannten Andreas Dadith, der sich viele Jahre zu den Unitariern gehalten hat. Unter dieser kirchlichen Zerrissenheit, die zagleich seine nächsten Verwandten spaltete, litt Chodkiewicz. Anf dem Reichstage za Lablin, der am 8. Mai 1566 angehoben and anf den ihn Weigel begleitet hätte, hörte er mit tiefstem Unwillen besonders von dem Anstarm der Baptisten. ${ }^{2}$ ) Alle staatliche and gesellschaftliche Ordnong schien ihm darch sie gefährdet. Gelegentlich seiner Ruckkehr ans Lablin blieb er eine Nacht in Stoklischkiø stddöstlich von Kanen and genob hier die Gastfreandschaft des gelehrten Wilnaer Stadtrogts Angustin Rotundus, den die Pest ans Lithanens Hantstadt vertrieben hatte. Einst (seit April 1539) hatte dieser in Wittenberg zu Lathers and Melanchthons Fußen gesessen, längst aber den Weg zar alten Kirche zurtickgefunden and sich wieder als deren eifriger Sohn gezeigt. In dem theologischen Gespräche, das sich beim Abendessen entspann, setzten er and zwei anweseude Mönche, nambafte Wilnaer Kanzelredner, Weigel hart zu. Aof Chodkiewicz machten ihre Einwendungen gegen die Reformation einen gewissen Eindruck, beim Aufbrach suchte er einen der Mönche zum Feldprediger für sein livländisches Heer za gewinnen. Als er im Mai 1567 nach Wilna zarlickkehrte und hier bei einem Gastmahl etliche nenerangssulehtige reformierte Prediger hart anfuhr, änBerte er za dem gleichfalls anwesenden Stadtrogt halb scherzend und halb wahr: "Hätte ich aus Stoklischki einen der Mönchsprediger erhalten, ich wäre wohl heate schon Papist." Nocb unsicherer warde er in seiner evangelischen Überzengung,

1) Seit dem 18. Dezember 1565 in Heidelberg; der Vater Martin Zborowski war am 25. Februar d. J. gestorben.

9) Vergl. Wotschke, Christoph Thretius, S. ol f.

3) Unfern Stoklischki hatte das Evangelinm in Rykonty, dem Besitze der Talwosz eine Stätte. Der lutherische Kastellan von Samogitien Nikolans Talwosz ( 1 1600) schickte seinen Sohn Adam, den späteren Hauptmann von Dünaburg ( +1628$)$ zum Studium nach Dentschland. Am 21. Juni 1579 ließ er sich in Königgberg einschreiben. 
als jetzt anf des Rotandus Betreiben ${ }^{1}$ ) anch Hosius Mahnschreiben an ihn richtete, ihm seine Konfession sandte and die katholische Kirche als den alleinigen Glanbenshort pries ${ }^{2}$ ).

Weigel war nicht der Mann, seinem schwankenden Herrn eine feste Stttze za sein. Schriften des Bischofs Lindanus and des Cölner Karthänsermönches Surius, die ihm Rotandus ans Wilna sandte, machten ibn selbst nnsicher and schwankend. Schon am 28. Januar 1568 konnte er ans Wenden nördlich von Riga, wohin er den Truppen des Hauptmanns von Samogitien gefolgt war"), schreiben ${ }^{4}$ ): „Libertas Lutherana pessumdabit Germaniam. Omnia sacra sunt prophanata et ad rudis plebeenlae notam atque voluntatem ditorta, at omnibus omnia liceant. Jam non amplius sustineo calvianus dici, etsi nec ante per omnia illi sectae addictus fai, tamen panlo melius de ea sensi qam nunc, abi video omnia plane sacra et humana violari et convelli. In hane finem semper collimarant aliqui Gastoldici Vilnenses religionis alioquin contemptores" ${ }^{\text {" }}$ ). Die Bitte um Übersendang weiterer Schriften, die Weigel am Schlnsse des Briefes an Rotundus richtete, fand nattirlich bei dem eifrigen Werberftir die alte Kirche willige Aufnahme. Bald erreichte der Wilnaer Vogt bei Chodkiewicz sein Ziel. Seine letzten Bedenken therwand der Nuntius Francesco Commendone'), der Anfang Dezember 1571 wieder nach Polen kam. Um ihn fur das evangelische Bekenntnis zartickzagewinnen, veranstaltete man eine Dispatation uber die Antorität der heiligen Schrift, an der anßer Chodkiewicz

1) Vgl, das Schreiben des Rotaddus aus Grodno vom 3. Angust 1567 an Hosins, E. S. Cyprian, Tabolarium ecclesiao Romanae S. 444f.

7) Opera Hosii II, S. 242 findet sich die Antwort des Hauptmanns von Samogitien auf das erste Schreiben des Kardiuals, das am 22. Juni 1567 in seine Hände gekommen war, S. 243 das zweite Sehreiben vom 80. Oktober 1567 ans Heilsberg.

7) Anfang 1568 belagerte Chodkiewicz vergeblich die Burg Ula, welche die Moskowiter anfern Polozk erbant hatten.

1) Vgl. E. S. Cyprian, Tabularium ecclesiae Romanae $578 \mathrm{ff}$.

5) Leider vermag ich nicht zu sagen, woranf Weigel hier anspielt. Der lithanische Kanzler Albert Gastold hat einst 1536 ff. Abraham Culvensis unterstützt. inm die Mittel zum Studium in Wittenberg and Italien gewährt. Aber von einem AnschluB dieser Familie an die Reformation ist nichts bekannt. Doch vergl, Corp. Rofor. $\mathbf{X}, 7$.

6) Vel. Cratian, de vita Commendoni S. $326 \mathrm{ff}$. 
sein Sohwager Andreas Zborowski, dazu die evangelischen Wojewoden ron Sendomir, Hohensalza, Brest, und viele andere teilnahmen. Der spanische Jesuit Franziskus Toletus ${ }^{1}$ ), der mit Commendone nach Polen gekommen war, verfocht die katholische Lehre, der bekannte Jakob Niemojewski, der so oft mit römischen Theologen die Klinge gekrenzt, unterstutzt von Stanislaus Drojewski ${ }^{2}$ ) die evangelische. Beide Parteien schrieben sich den Sieg $\mathbf{z}^{8}$ ). Jedenfalls konnte die Dispatation Chodkiewicz Entschlob nicht rtickgängig machen. Fortan bekandete er regen Konvertiteneifer. Bei der Krönung König Heinrichs arbeitete er z. B. mit allem Nachdruck im Sinne des Hosins, um den von der Warschaner Konfoederation geforderten Eid des Königg auf die pacta conventa, der

1) Toletus starb als Kardinal in Rom am 14. Sept. 1596.

7) Anch Drohojowski genannt. Dieser treu erangelische Kastellan von Przemjel, hatte am 20. Okt. 1542 die Leucorea bezogen, war dann nach Italien, 1547 nach Zürich und Straßburg gegangen. Mit Flacius, der ihn un Material für seine kirchengeschichtlichen Arbeiten bat, stand er in Verbindnng (vgl. seinen Brief vom 6. Juni 1556 bei Wotschke, Francesco Stancaro S. 33). Zar Dracklegang der polnischen Bibelübersetzung stellte or Geld zur Verfügnng, auf seinem Erbgute Drobojow und in Jacmierza (beide Orte liegen bei Sanok in Galizion) führte er die Reformation ein. Einige Jahre war er ein Gönner und Schutzherr Stancaros. Er starb bald nach 1580. - Der Matthis Stanislaus Drohojowski, der seit dem 21. Sept. 1607 in Heidelberg studierte, in demselben Jahre mit seinem Bruder Johann, den wir seit dem 16. Ort, 1611 in Leyden sehen, auch die Marburger Hochschule bezog, der Stanislaus Dr., der mit seinem Bruder Andreas seit April 1617 in Herborn stadierte, hier 1619 eine Disputation de prndentia et iustitia reröffentlichte, waren wohl seine Enkel, die Söhne des um die evangelische Kirche verdienten Kastellans von Sanok Johann Drohojowski, Der letztgenannte Stanislans Dr. hat 1645 das Thorner Bekenntnis unterschrieben. Sein 1624 geboreaer Sohn Stanislans studierte seit dem 5. Juli 1644 in Leyden, sein Sohn Andreas seit 1651 in Frankfurt Das Thorner Gymnasium besuchten seit 1648 die Brüder Christoph und Wladislaus Dr., erstererer ist der spätere Przemysler Bannerträger, der manche Synode, 1682 die zu Radzienczyn im Labliner Land, geleitet hat.

9) Nähere Nachrichten tiber dies Religionsgespräch gibt ein Brief des Gratian an den znm Katholizismus übergetretenen Nikolans Tomicli deu Sohn des Gnesener Kastellans Johann Tomicki, aus Warschau von 2. April 1572. Als Trumpf gegen Niemojewski, der sich des SiegłB rühmte, veröffentlichte die Gegenseite den Brief poluisch and lateinisch atn 18. Angust 1580. Vgl. Scriptores rerum Polonicaram VII, 225. 
Religionsfreiheit verburgte, zu verbindern oder abzusch wächen ${ }^{1}$ ). Von den Gotteshänsern, in denen Chodkiewicz den römischeu MeBgottesdienst wieder anfrichtete, seien die Kirchen in Martynow (in Wolhynien unfern Luzk) und Hnezna (anfern Wolkowischki), in der 1588 Chodkiewicz' Gattin ihre letzte Rabestätte fand, genannt $t^{2}$ ). Chodkiewicz selbst starb schon 1578 $)$. Von erangelischer Seite sind ihm noch 1574 ron Nikolaus Rej, dem polnischen Hutten, und 1577 vou Bernhard Gorecki Schriften zugeeignet worden.

Trotz langen Schwankens blieb Weigel schlieBlich doch der Reformation trea. Dem Beispiele seines Herrn folgte ernicht. "Mnnera, dum vivo, taa, Lencoris alma, tenebo, Quaque decet memori mente fideque colam", hatte er 1561 gelobt. In der Tat brannte die Liebe zur Reformationsstadt in seinem Herzen. Nach des Chodkiewicz Übertritt kehrte er nach Wittenberg zurttck. Hier sehen wir ihn 1573. Jn das Stadentenalbum 4 ) des Clandius Textor ans Savoyen, der am 15. April 1564 sich an der Leacorea hatte einschreiben lassen, trägt er Matth. 5,10, dazn lateinische und griechische Verse tber diesen Spruch ein. Es ist das letzte, was ich ther ihn ermitteln konnte.

I. Georg Weigel an Herrog Albrecht.

Goadt, fridt vnd alle heilsame wolfart dorch Christum neben erpietung meiner alzeytt schuldigen, vnderthenigen, willigen vnd gehorsamen dienste vnd demtitigem gebett $\mathrm{zu}$ gott benor. Gnedigster furst vnd herr. Ioh bin den 24. Juni, an S. Johannis des tänfers tag, gott lob frisch vud

1) Am 12. Dezember 1573 hatte Hosins deshalb an ihn geschrieben. Hosii opera II, 374ff.

2) Das Gotteshans in Szklow am Dniepr, eine der ostlichsten reformierten Kirchen im Reformationsjahrhnndert hat sein zweiter Sohn Alexsnder, der Wojewodo ron Troki, katholisiert.

3) Februar 1578 gewann er noch den Rigaer Arzt Zacharias Slopius. den Brader des Kottbuser Stadtachreibers Hieronymus Slopius fär seine Dienste. Cichocki schreibt in den colloquia Osieconsis von Chodkiewicz: „Vir sine controversia magnus, quem vulgus terrorem improborum hominum vocare consueverat, omnes fere haereses antea perragatus tandem levitatem inconstantiamque flnctuantium dogmatum detestatus toto animo catholicam amplexus est religionem, in qua toenda adeo profecit, ut palam solidis rationibus assertores istos novi erangelii impietatis convinceret."

4) Im Besitze der Lutherhalle in Wittenberg. 
gesundt gen Witeberg ankhomen vnd bab e. f. d. brieff eim jedlichen jn sonderheytt ond D. Maiori brieff ond die $100 \mathrm{ft}$. selbs treulich vnd fleiBig nach e. $f$. $d$. gnedigem beuelch uberantwortet, welche alle zn jrer zeytt sich gegen e.f. d. vndertheniglich bedanckhen werden, insonderbeit $\mathrm{D}$. Maior, welche. mir sagt, jch khäme jm daramb gantz gelegener zeytt, weyll er morgen, dab ist den andern tag meiner ankhonft, seiner tochter, welche er eim jungen magistro, ') so nun jura studiret, verheirat, hochzeytt zu halten gedacht were. Herr D. Jonas war nit anbeim, sonder wie jch von seim gemahel vod letzlich von jme selbs verstanden, jn churftirstlichen vou Sachsen geschefften, welcher e. $f$. d. brieff mit hoher frende gelesen vnd sich meiner ankbnnft zn e. f. d. mit mir tröstlich erfreuet vnd wie sein branch e. f. d. gnedigen willen, lust vod lieb zum wort gottes mit merern worten exaggerirt vnd confirmirt hat, also daB jeh, weiB goth, je lenger je mer vou hertzen beger, einer solchen theologischen furstlichen personen, welche incorruptam evangelii vocem et ipsins ministros lieb vod werdt helt, ernert rnd promoniert, jn vnderthenikheitt neher za sein. Vnd khan auff e.f. d. mit grandt vnd warheit der spruch Esaiae 49. wol gezogen werden: „erunt reges nutritii tui et reginae nutrices tuae." Also khan vnd will dan der fromme gott solche christlichen frommen regenten mit frolichem mundt anreden psalmo 81.: „ego dixi, dii estis et filii excelsi omnes".

Hiemit, gnediger furst vnd herr, schickb jeh e. f. d. zwey exemplaria der gehaltenen gedechtnus Philippi, dariı die zwen vers stehen. Mich rewet es offt, $\mathrm{daB}$ jch von e. f. d. khein anleitang brieflein an meine herren von Närnberg (wie mir wol za than gebthrt hett, aber auß vergessen vnderlassen) vndertheniglich begert vnd versucht habe, so hetten sie desto mer vrsach gehabt, mich maiori humanitate ét liberalitate von jnen $z$ lassen. Pitte hiemit den trewen lieben gott, er wolle e. f. d., derselben christlich lieb gemaht vnd junge hersehafft jn langwiriger gesundtheit vnd gluckseligem regiment gnediglich erbalten vad von allem tubell leibs vnd seel bewaren. Amen. Thue mich derselben e.f. d. jn vnderthenickbeit ganz vnd gar ergeben. Datnm Witeberg, den 30. Junii anno Christi 1561. E. f. d. vndertheniger vnd geborsamer Georgins Vueigelius.

\section{Georg Weigel an Martin Faber.}

Percupio abs te cognoscere, hamanissime mi d. M. Martine, quam feliciter Noribergam veneris et qua etiam nunc valetadine quove successu fruaris. Gratulor tibi, si conditionem te dignam et tranquillam consecutus. es. E'i

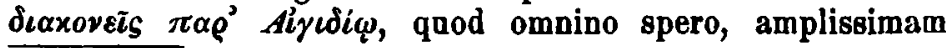

1) M. Joh. Purgold ans Eisenach, 
habes occasionem coutrabendi amicitiam cum Nentauichio, viro optimo fratre mihi carissimo, too favore et benerolentia dignissimo. Hanc ipsam, ne quid amicitiae nostrae desit, in meum locam interea statno, qui sua morum suavitate et conditione facile efficiet, at ipsins nomine et me arctins sis complexurus. Sic ofticiosus est, at gandeat sibi dari occasionem de quoque bene merendi, tam fidelis, ut proximi magis salutem quam suam curet, adeo candidus et apertas, at dissi-

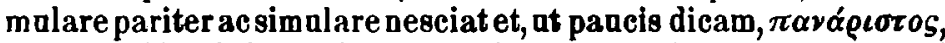

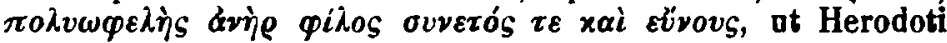
voce utar. Hane et meo et me ipsins nomine amabis, sumus enim idem corpus. Hic omnia adhuc salva sunt. Ad 8. Augusti diem designati sunt magistri numero $33^{1}$ ), in quibus et nostri Gronus ${ }^{2}$ ) et Helmus ${ }^{8}$ ) erant. M. Schoppius 28. die Angusti suas eelebravit nuptias satis solemniter, ad quas nos Noribergenses ferme omnes convenimus. Die Augusti 14. obiit Elisabetha, filia D. Maioris nata media. Dispntatio inter M. Victorinum et Illyricum interrapta est morte filioli principis Saxoniae Janfriderici natn maioris. Doctor Maior paulo ante mortem filiae aegrotare coepit et hue usque graviter decabuit, nuuc melius habet, pro quo deam oramus, ne suam naviculam omnibus his gubernatoribus destituat; sed eam ipsam regat et doceat et subinde alios aliis nancleros subiongat. Ramuscalus hic est regem Galliae expediisse legatos ad caesarem de ablegandis nostris ad se theologis, ot cum ipsins conferant. Ita enim homines flagrare purioris, si quae praeter asitatam sit, doctrinae studio, at si diatias recuset, periculam sit de tomaltis. Plara alias. Bene vate. Vuittebergae raptim 1560 die Septembris 27. $)$

\section{Matthăus Motzarus an den Kanzler Hans von Kreitzen.}

Quamquam, magnifice domine, nallis a me stadiis lacessitus, maltis tamen officiis a Taa M. D. sum cumalatus, pro quibus tantnm me Tuae M. D. debere intelligo, at nallis offieiis, nulla opera, labore industriaque mea posse videar satisfacere. Quae quoniam ex aequo reponere non possum, referet M. D. Tuae hio, qui pietatis officia malto cam foenore solet remetiri. Sic etiam per sexenniam Alberti olim principis beneficiis usus sum, enins etiam munificentia ad culmen et fastiguam eraditionis in celebri hac academia Regiomontana

1) Vgl. Köstlin, die Baccalaurei and Magistri der Wittenberger philos. Faknität 1548-1560 S. 23.

7) Melchior Gruen, seit Februar 1555 in Wittenberg, später Professor der Logik in Wittenberg.

7) Melchior Helm, seit Augast 1557 in Wittenberg.

4) Dieser Brief ist entnommen dem Codex Gothanas chart. A. 123, B1. 277 . 
aspiravi ${ }^{1}$ ), et abi doctrinae mediocris quandam cognitionem mihi comparaveram, alio me consensu einsdem principis obligans me ad reversionem chirographo contali," at Tua M. D. hand dabie habet in recenti memoria, praesertim cam per Tuam M. D. cam ill. prineipe de impetranda renia egi. Quoniam antem ultra terminum in chirographo ${ }^{2}$ ) expressum in peregrina rel exterranea natione moror, videtur et fides apnd me nanfragiam fecisse et fructus ingenii mei non in obsequia suae celsitudinis sed aliunde divertisse, ideo non immerito macula ingratitudinis, quae mihi semper invisa detestataque est, videor nunc notandas ... Paucis attigi in literis meis ad ill. principem, quibus rationibus adductos peracta peregrinatione in regno Poloniae moror, nimiram quod mihi cuiusdam gymnasii curam contra propriam voluntatem a quodam magnate regio commissa est, quam iam deponere vellem, si ill. principis literae, quales in literis ? ad suam celsitudinem expressi, advolabont. Quam obrem Tuam M. D. submisse rogo, ut cum sua celsitudine hoc in meo negotio ita agat, quo et sim apud suam celsitudinem excusatus, quod dintius insto in peregrina natione ultra

1) Hotzarus stammte ans Lyck, hat in Königsberg stadiert un 1566 eine Rede de excubiis angelorum dem Herzog Albrecht gewidmet. Ende 1571 gewann ihn der Radziwillsche Hauptmann in Klezk, Hieronymus Makowiecki, der Frühjahr 1563 mit dem jungen Nikolaus Christoph Radziwill nach Straßburg gezogen war, damals von dem bekannten Unitarier Simon Budny gebeten war, Bnllingers Ansicht über das zwischen der griechischen und römischen Kirche strittige "filioque einzuholen, der September 1563 von l'übingen über Stuttgart nack Lithanen zorïckging, zum Leiter der Schule in Klezk (zwischen deu Eisenbahnknotenpunkt Baranowitgchi und Slazk). Innerhalb der vor den unitarischen Predigern Thomas Falkonius and Simon Badny geleiteten Gemeinde hat er als lntherischer Lehrer gewirkt. Doch nur kurze Zeit. Die noch 1572 ans Leipzig zarückkehrenden Albrechi und Stanislans Radziwill richteten wohl schon im nächsten Jabre den Katbolizismus in Klezk wieder anf.

2) Vgl. oben S. 35 .

3) An den Herzog Albrecht Friedrich hatte Motzarng an dem. relb'en Tage geschrieben: „Post longam iactationem fati, nbi me fortuna tandem ex variis periculis emersisset, in optatum portum detulisset, duxi mibi in Polonia paululum respirandum. Et interea dam in auka regis Polonornm versur, opinione fortasse alicuins in me eruditionir quidsm de proceribus Hieronimns Makovietius apnd regem effecit, ut me suo gymnasio Klecensi praeficeret, quod, ne roluntati regise videar cessisse, nefas mihi detractare indicavi concessique verum tantum in annum docendi munns, quod iam invante deo die natalitii Christi conficiam deponamque. Veram ne contra propriam voluntatem petitionibus eiusdem dni Hieronimi Makovietii, capitanei Klecensis, viri sane 
terminum in chyrographo datam maseo, et simal hae literae, ut sim nimiram fonctione bac scholastica vacuns, mibi a sua celsitudine elargiantur, sine quibus deserere conditionem sine magna molestia et commotione eins, qui mibi ean iniunxit, non possum.

Significavi etiam suae celsitudini brevibus, qualis esset rei publicae Polonoram status et quod ex tribas die epiphaniarum fieri deberet electio nnaque ex his ant fratrem caesaris aut principem nostram Prassiae aut dacis Moscorum filinm proceres dominii in regnom sabstituere vellent. Exposai simal suae celsitudini menm consiliam, quo pacto sua celsitudo prae ceteris potiri regno Poloniae poterit, quod procul dabio ex literis meis ad saam celsitudinem hand obscare $M$. D. T. perspiciet. $\mathrm{Si}$ antem $\mathrm{M}$. D. $\mathrm{T}$. rationes consilii mei non omnino displicuerint, pergratissimnm mihi T. M. D. fecerit, si principis nostri animnm M. D. T. eo inflectat, at huic consilio assentiat. Ego cum his proceribng, quos in literis meis ad suam celsitudinem memini, ita cantissime invante deo rem agam loco. tempore occasioneque idouea, at nulla inde suspitio nascatur, hanc suae celsitudinis voluntatem fuisse. Si ill.princeps consilio acquievit, rogo $T$. M. D., ut quoque efficiat, ut sua celsitudo, saltem duo equi sant mihi, alios duos, vestimenta, pecanias suppeditet, solomodo at in aala horam procernm regni, in quibus cardo totias regni vergatur et cum quibus etiam mibi res erit, non inferior in omnibus ceteris eornm aulicis appaream. Hae vero impensae, si res ex voto ceciderit, quanto cum foenore revertantur, luvante deo eventog ipse ostendet. Sed hac in re maturandam est, at habeam spatium, quo in procerum animos, insinuem, at mibi rationes suaram sententiarum communicent, et hac nacta occasione conveniente loco et tempore ad amplificandas principis nostri landes nec consilium nee studium meum deerit.

Tua M. D. non gravetur mihi perseribere, qualis sit status reipablicae Prussiacae post ademptum nobis principem Albertam seniorem. Incerti euim rumores de ea apud nos vagantar, quare id certissimum existimabo, quicquid ex M.D.T. cognovero. Interim me meaque omnis generis obsequia M. D. Toa sibi commendata habeat. Datae ex Klecko 12. Norembris anno 1572. M. D. T. famulas obsequentissimas Matthaens Motzarus.

eruditi, succumbam et in sequentem annum onas et molestias gymnasii sustineam, submisse T. Celsitudinem obsecro, nt huic rei T:a Celsitudo clementer occurat." Der Herzog wöchte ibn sls seinen Entertanen nnd Stipendiaten heimrafen und bei Makowiecki seine Bntlassung erwirken. Ans Dank würde er bei der bevorstehenden Königswahl die Stimmen der polnischen Großen anf ihn lenken. 